\title{
Itinéraire d'un inquisiteur gâté : Ponce Feugeyron, les juifs et le sabbat des sorciers
}

\section{Madame Martine Ostorero}

\section{Résumé}

Dans la première moitié du XVe siècle, l'inquisiteur franciscain Ponce Feugeyron est mandaté par le pape Alexandre $V$ pour agir tant contre les juifs et les judaïsants que contre les sectes d'hérétiques et de sorciers. Son rayon d'action couvre tout le Sud-Est de la France, d'Avignon au duché de Savoie en passant par le Dauphiné. On examine ici l'hypothèse qu'il pourrait être l'auteur des Errores gazariorum, l'un des plus anciens textes présentant l'imaginaire du sabbat, écrit dans le val d'Aoste vers 1436.

\begin{abstract}
Itinerary of a spoiled inquisitor: Ponce Feugeyron, the Jews and the witches' sabbath - A Franciscan living in the first half of the fifteenth century, Ponce Feugeyron was an inquisitor whose activities spread over Jews, heretics and witches. He covered an area wich included nearly all of Southeastern France, from Avigon throught the Dauphiné to the duchy of Savoy. He might also be the author of one of the very first texts mentioning the witches' sabbath, namely Errores gazariorum (errors of the "Gazari »), an anonymous treatise written in the Aosta Valley around 1436.
\end{abstract}

\section{Citer ce document / Cite this document :}

Ostorero Martine. Itinéraire d'un inquisiteur gâté : Ponce Feugeyron, les juifs et le sabbat des sorciers. In: Médiévales, $n^{\circ} 43,2002$. Le bain : espaces et pratiques. pp. 103-117.

doi : 10.3406/medi.2002.1561

http://www.persee.fr/doc/medi_0751-2708_2002_num_21_43_1561

Document généré le 16/10/2015 
Martine OSTORERO

\section{ITINÉRAIRE D'UN INQUISITEUR GÂTÉ : PONCE FEUGEYRON, LES JUIFS ET LE SABBAT DES SORCIERS}

L'objectif de ces quelques pages est de reconstituer la trajectoire d'un inquisiteur, son rayon d'action et ses préoccupations majeures. En effet, depuis peu les recherches prosopographiques sur les inquisiteurs ont montré tout leur intérêt. Dans la plupart des cas, on constate que derrière l'institution de l'inquisition se cachent des personnes ancrées dans un vécu culturel et intellectuel, dotées d'expériences propres, et que l'activité inquisitoriale dépend largement des personnes qui la mènent ${ }^{1}$.

L'inquisiteur choisi ici n'est pas anodin: le franciscain Ponce Feugeyron effectue un riche parcours dans la période cruciale de la première moitié du $\mathrm{XV}^{\mathrm{e}}$ siècle, au moment de l'émergence du fantasme des sectes démoniaques. Par ailleurs, à l'occasion d'une relecture des textes parus dans L'Imaginaire du sabbat ${ }^{2}$, j'aimerais poser l'hypothèse qu'il puisse être l'auteur du texte anonyme des Errores gazariorum, l'un des premiers témoignages sur le sabbat des sorcières, daté de 1436 environ - ou tout au moins très proche de son contexte d'élaboration.

1. Citons pour exemple l'article très fouillé de B. ANDENMATTEN et K. UTZ TREMP, « De l'hérésie à la sorcellerie : l'inquisiteur Ulric de Torrenté OP (vers 1420-1445) et l'affermissement de l'inquisition en Suisse romande », Revue d'histoire ecclésiastique suisse, 86 (1992), p. 69-119, ainsi que Les Inquisiteurs. Portraits de défenseurs de la foi en Languedoc (XIII'-XIVe siècle), L. ALBARET éd., Toulouse, 2001.

2. L'Imaginaire du sabbat. Édition critique des textes les plus anciens (1430c.1440c.), réunis par M. OStorero, A. PARAVICINI BAGLIANi et K. UTZ TremP, en collaboration avec C. CHÈNE, Lausanne, 1999 (Cahiers lausannois d'histoire médiévale, 26). Voir le dossier "Aux sources du sabbat", dans le précédent numéro de Médiévales, p. 153-176. 
En effet, dans mon commentaire des Errores gazariorum, j'avais suggéré que le texte pouvait avoir été rédigé dans la région du val d'Aoste. Cette proposition se fondait sur deux indices: un nom de personne - Jean de Stipulis, soit Jean d'Étroubles, un village situé sur le versant sud du Grand-Saint-Bernard - et un nom de lieu, Chambava, que l'on peut identifier avec Chambave, une localité valdôtaine où a été brûlée une sorcière. Or cette femme, désignée dans les Errores sous le nom de Jeanne Vacanda, a maintenant été identifiée avec Jeannette Cauda (Johanneta Cauda), le nom ayant été mal transmis dans les manuscrits du traité anonyme. Les frais de son exécution ont été retrouvés par Ezio E. Gerbore dans les comptes de la châtellenie de Cly : ils attestent qu'en 1428 Jeannette Cauda a bien été brûlée, comme le rapportaient les Errores, sur la place de Chambave, le jour de la SaintLaurent, fête patronale du dit lieu ${ }^{3}$. L'hypothèse de l'origine valdôtaine du texte se trouve donc maintenant confirmée. Cette région connaît des procès de sorcellerie dès 1434 au moins et des recherches encore inédites viennent de mettre à jour un important dossier de procédures ${ }^{4}$. La précocité du phénomène peut être mise en relation avec la présence à la tête du diocèse d'Aoste de l'évêque Georges de Saluces (1433-1440) : celui-ci joue un rôle important dans le début des chasses aux sorciers, tant dans le diocèse d'Aoste que dans celui de Lausanne où il est ensuite déplacé en 1440. L'inquisition est placée entre les mains des franciscains. Après Barthélemy Revottini en 1428 et 1430, on trouve un autre représentant du tribunal d'inquisition en la personne d'un magister Poncius, frère mineur. Dans une note de l'édition des Errores gazariorum, j'avais proposé d'identifier ce «maître Ponce» avec Ponce Feugeyron, inquisiteur franciscain, à qui est adressée une célèbre bulle d'Alexandre V en 1409. Le document, retrouvé par Silvia Bertolin, confirme qu'il s'agit bien de lui, même si son patronyme est transmis sous la forme Frugeronis ${ }^{5}$. Or, Wolfgang Behringer, dans un article récent ${ }^{6}$, poursuit la réflexion en suggérant - sans étayer son propos -

3. Voir E. E. Gerbore et B. ORLANDONI, Il castello di Cly. Storia ed evoluzione di un castello valdostano, Aoste, 1998.

4. Outre les recherches encore inédites d'Ezio E. GERBORE, voir S. BERTOLIN, L'Inquisizione in Valle d'Aosta tra XV et XVI secolo, tesi di laurea Università degli studi di Milano, Facultà di Giurisprudenza, anno accademico 2000-2001.

5. M. OSTORERO et alii, op. cit., p. 330-334. Voir S. BERTOLIN, op. cit., p. 87 et 227. Sur les différentes formes du patronyme, voir infra $\mathbf{n}$. 7. Selon $\mathrm{E}$. Gerbore, d'autres documents attestent la présence de Ponce Feugeyron dans la vallée d'Aoste et son activité d'inquisiteur.

6. W. BEHRINGER, « How Waldensians Became Witches : Heretics and Their Journey to the Other World », à paraître dans les actes du colloque Demons, Spirits, Witches. Popular Mythology and Christian Demonology (7-9 octobre 1999, Budapest, Hungarian Academy of Sciences). 
que Ponce Feugeyron puisse être l'auteur des Errores gazariorum. Je propose ici de tester cette hypothèse en retraçant l'itinéraire de cet inquisiteur franciscain entre 1409 et 1438 .

\section{La bulle de 1409 et ses confirmations}

Avant 1409 et la bulle d'Alexandre V adressée à Ponce Feugeyron, on sait peu de choses du personnage. On peut supposer qu'il naquit vers 1380 et qu'il suivit sa formation chez les franciscains au tournant des années 1400, probablement à Avignon, car c'est au couvent de ce lieu qu'il semble se rattacher par la suite. En août 1409, il est désignć comme maître en théologie et inquisiteur de Provence ${ }^{8}$. Le 30 août 1409 , soit moins de deux mois après son élection au siège pontifical, Alexandre $\mathrm{V}$ proclame la bulle qui nous intéresse ici. Nous sommes au moment culminant du schisme, puisqu'Alexandre $\mathrm{V}$ a été élu le 26 juin 1409 après les dépositions de Grégoire XII et Benoît XIII par le concile de Pise : la papauté a donc trois têtes, chacune reconnue par des partis différents. Dans sa bulle, Alexandre V fait d'emblée allusion au

7. L'orthographe Feugeyron a été retenue ici en raison de sa fréquence d'apparition. C'est aussi la forme employée dans les bulles pontificales (Archivio Segreto Vaticano, Reg(istra) Lat(eranensia) $136, f^{\circ} 177 r^{\circ}-178 r^{\circ}$ et $279 v^{\circ}-280 r^{\circ}$; Reg. Lat. 189 , $\mathrm{f}^{\circ} 267 \mathrm{v}^{\circ}-268 \mathrm{v}^{\circ}$; Reg. Lat. 190, $\left.\mathrm{f}^{\circ} 97 \mathrm{r}^{\circ}-98 \mathrm{v}^{\circ}\right)$. Les variantes Fougeyron, Frugeronis, Fergonis, Fongerons ou encore Sengeronis ont été écartées. La provenance du personnage reste difficile à établir; elle pourrait être savoyarde ou piémontaise : on trouve un Pietro di Fongereto, abbé de Saint-Michel de la Cluse (Savoie) à la fin du XIve siècle. Voir B. Galland, Les Papes d'Avignon et la Maison de Savoie (1309-1409), Rome, 1998 (Collection de l'école française de Rome, 247), p. 392-393.

8. Le 12 août 1409, Alexandre $V$ charge Ponce Feugeyron de conférer la maîtrisc en théologie à Guillaume Viviani et à Pierre Fabre de l'ordre des frères mineurs. Luimême est qualifié de maître en théologie (C. EuBEL, Bullarium franciscanum, vol. 7, Rome, 1904 , p. $412, n^{\circ} 1180$ et J. M. VIDAL, Bullaire de l'inquisition française au $X I V^{e}$ siècle, Paris, 1913, p. 487-488 et n. 1). Pierre Fabre ou Fabri sera nommé inquisiteur en 1419 en Dauphiné, pour agir contre des vaudois, probablement avec Ponce Feugeyron, puisque tous deux furent confirmés dans leur fonction par Eugène IV en 1434. Voir P. Paravy, De la chrétienté romaine à la Réforme en Dauphiné, Rome, 1993 (Collection de l'École française de Rome, 183, vol. 2, p. 975) et C. EUBEL, op. cit., $\mathrm{n}^{\circ} 1181$ et 1421 . Fabri est inquisiteur lors des procès de Thomas Bègue et d'autres hérétiques en 1436 à Exilles, puis il agit en 1437 contre l'hérétique Étienne Bleyn. Voir J. MARX, L'Inquisition en Dauphiné. Étude sur le développement et la répression de l'hérésie et de la sorcellerie du XIV" siècle au début du règne de François ler, Paris, 1914 (Laffite Reprints, Marseille, 1978), p. 214-217, n०8 et 9. En 1432, Fabri écrivait d'Embrun au concile de Bâle pour s'excuser de ne pouvoir se rendre auprès des pères du concile à cause des infirmités corporelles qui l'affligeaient et de l'indicible pauvreté qu'il endurait, n'ayant jamais reçu un denier de l'Église et ne recevant de gages de personnes (ibid., p. 65). 
contexte du schisme, puisqu'il enjoint Ponce Feugeyron d'agir contre ceux qui s'opposent à son élection et aux décisions du concile de Pise. Or, le mandat d'action de Feugeyron a effet notamment en Avignon, dans le Comtat Venaissin et en Provence, soit dans les terres même de Benoît XIII, le pape de l'obédience avignonnaise, et à ce titre le premier adversaire d'Alexandre $V$. On voit comment Alexandre $V$ cherche à fonder son autorité en envoyant un inquisiteur combattre les schismatiques sur le terrain de son rival, très peu de temps après que ce dernier a été contraint de fuir la cité des Papes.

En dehors de ce mandat contre les adversaires d'Alexandre V, la bulle de 1409 contient cinq articles. Le premier dénonce le fait que, dans plusieurs régions, certains juifs et chrétiens ont inventé de «nouvelles sectes » assorties de «rites prohibés » et qu'ils font du prosélytisme contre la foi chrétienne. Le deuxième article précise que chrétiens et juifs, sorciers, devins, invocateurs de démons, enchanteurs, conjurateurs et augures superstitieux, usant d'arts criminels (nefarius) et prohibés, pervertissent le peuple. Au troisième article, il est question des juifs qui tentent de récupérer leurs anciens co-religionnaires récemment convertis au christianisme. Le quatrième article déclare que des juifs cherchent à diffuser le Talmud et d'autres livres erronés, se détachant même des textes de la loi mosaïque (atque etiam in sua lege Mosayca multipharie deficentes). Enfin, dans le dernier article de la bulle de 1409, il est question des chrétiens et des juifs prétendant que l'usure n'est pas un péché et qu'il est permis de prélever dix pour-cent pour un prêt ${ }^{9}$.

La bulle est dirigée avant tout contre les juifs, et en particulier contre les judaïsants et les récents convertis retournés au judaïsme, contrairement à ce que l'extrait publié par Joseph Hansen donnait à penser, puisque l'historien allemand, dans son anthologie de textes sur la sorcellerie médiévale, n'avait pris en compte que les articles concernant les nouvelles sectes et les sorciers invocateurs de démons ${ }^{10}$. La récente réédition

9. Sur la question de l'usure, voir le dernier ouvrage de J. SHATZMILLER, Shylock revu et corrigé : les juifs, les chrétiens et le prêt d'argent dans la société médiévale, Paris, 2000.

10. J. HANSEN, Quellen und Untersuchungen zur Geschichte des Hexenwahns und der Hexenverfolgung im Mittelalter, Bonn, 1901 (reprint Hildesheim, 1963), p. 16-17. Joseph Hansen n'a repris que partiellement la bulle éditée jadis par L. WADDING, Annales Minorum seu historia trium ordinum a Sancto Francisco institutorum, Rome, 1734, t. 9, p. 327-329, puis par C. EuBEL, op. cit., p. 413-414 $n^{\circ} 1181$. Cela a passablement biaisé la compréhension du texte, d'autant plus que la plupart des historiens de la sorcellerie ne se référaient qu'à la version de Joseph Hansen. 
de la bulle par Shlomo Simonsohn ${ }^{11}$ permet enfin de redécouvrir le texte dans son intégralité et de réinterpréter l'idée de novas sectas dans le contexte particulier de ce début de $\mathrm{XV}^{\mathrm{e}}$ siècle, au moment de l'émergence de l'imaginaire du sabbat des sorciers. Soulignons-le, la bulle vise en premier lieu les juifs : elle condamne l'usure, l'usage du Talmud et des autres livres hébreux, ainsi que la magie qui serait employée dans des cercles juifs ; elle réprime les judaïsants et ceux qui font du prosélytisme pour rejudaïser les nouveaux chrétiens. Ces condamnations ne sont pas des nouveautés; elles s'inscrivent dans un mouvement de contrôle des juifs dans leurs rapports avec les chrétiens, commencé dès le concile de Latran IV en 1215 qui exige le port d'un signe distinctif pour les juifs. Depuis la bulle Turbato corde de Clément IV en 1267, les inquisiteurs sont habilités à agir dans ces différents domaines, dans la mesure où cela implique des conséquences pour les chrétiens, qui risqueraient d'être corrompus par les juifs ${ }^{12}$. Ainsi, et comme le théorise Bernard Gui dans la cinquième partie de sa Practica Inquisitionis (1314-1324), les juifs convertis au christianisme qui retourneraient au judaïsme, ut canes ad vomitum, sont considérés comme apostats et leur cas relève de la justice de l'Église ${ }^{13}$. Ce climat de suspicion est clairement perceptible dans le Fortalicium Fidei du franciscain espagnol Alphonse de Spina qui, vers 1460 , prônera l'instauration d'une inquisition contre les judaïsants; il sera entendu en 1478, au moment où Sixte IV fondera l'inquisition dans la péninsule ibérique ${ }^{14}$. La question du Talmud, évoquée dans le quatrième article de la bulle, a posé problème pour les chrétiens : accepté à l'origine comme un ouvrage propre aux juifs et bénéficiant de ce fait d'une cərtaine tolérance, le Talmud en vient à être considéré comme un instrument idéologique dangereux pour la foi chrétienne et comme un livre hérétique; à la suite des importantes disputes et controverses parisiennes (1240-1244), le

11. S. Simonsohn, The Apostolic See and the Jews. Documents: 1394-1464, vol. 2, Toronto, 1989 (Studies and Texts, 95), p. 658-661.

12. A. FoA, "The Witch and the Jew : Two Alikes that Were Not the Same », dans From Witness to Witchcraft. Jews and Judaism in Medieval Christian Thought, J. COHEN éd., Wiesbaden, 1996, p. 361-374, sp. p. 365.

13. S. Simonsohn, The Apostolic See and the Jews. History, vol. 7, Toronto, 1991 (Studies and Texts, 109), p. 234-238. S. GRAYZEL, The Church and the Jews in the XIIIth Century. A Study of their Relations during the Years 1198-1254, based on the Papal Letters and the Conciliar Decrees of the Period, t. II, New York, 1989, p. 12-22. J. COHEN, The Friars and the Jews. The Evolution of Medieval Antijudaism, Ithaca-New York, 1982. L. I. NEWMAN, Jewish Influence on Christian Reform Movements, New York, 1925.

14. J. HANSEN, op. cit., p. 145-148. 
Talmud est condamné par Innocent IV en 1244 et en 1248, puis par Jean XXII en $1320^{15}$. Quant au problème de la stigmatisation de la magie juive, elle apparaît sur le devant de la scène lors du prétendu complot de 1321 entre les juifs et les lépreux, soupçonnés d'avoir empoisonné les puits avec des poudres noires données par les juifs ${ }^{16}$. Quelques années plus tard, en 1326, Jean XXII, dans la bulle Super illius specula condamne l'usage de la magie, dans la mesure où elle implique des liens avec les démons, et déclare l'inquisition compétente en la matière; les juifs ne sont toutefois pas mentionnés expressément.

Dans la bulle de 1409, les chrétiens sont associés aux juifs pour l'usure, les pratiques magiques ou de sorcellerie, et surtout pour l'invention de nouvelles sectes qui semblent réunir des individus des deux observances religieuses; et cela dans un contexte où, effectivement, il est fort possible qu'il y ait eu des contacts entre ces deux communautés, au moment où les juifs ont été chassés du royaume de France et ont trouvé refuge notamment en Avignon et dans le Comtat Venaissin, territoires du Siège Apostolique. Le début du XVe siècle connaît un afflux important de juifs en Avignon, au point que la communauté juive de la cité doit agrandir son cimetière en $1414^{17}$. Le pape, et avec lui l'inquisiteur, semblent donc percevoir des risques dans cette promiscuité excessive qui pourrait conduire à des déviations syncrétiques ${ }^{18}$. De là cette peur de nouvelles sectes, réelles ou imaginaires, qui auraient inventé de dangereux rituels. C'est là la grande nouveauté de la bulle.

Mais, soulignons-le, la bulle ne parle en aucun cas de ce qui pourrait ressembler à un sabbat : nulle mention de cérémonies nocturnes, de rites orgiaques ou cannibales, de vols magiques, etc. De plus la mention des « nouvelles sectes » n'est pas liée à l'article concernant la magie et la sorcellerie; les deux choses sont encore bien différenciées, et on ne peut voir dans la bulle d'Alexandre V de 1409 la désignation d'un sabbat des sorcières. Par contre, il s'y trouve des éléments qui mèneront certaine-

15. A. TUILLIER, « La condamnation du Talmud par les maîtres universitaires parisiens, ses causes et ses conséquences politiques et idéologiques », p. 59-78, sp. p. 75 , dans Le Brûlement du Talmud à Paris: 1242-1244, G. DAHAN éd., Paris, 1999. E. A. Synan, The Popes and the Jews in the Middle Ages, New York, 1965, p. 131.

16. C. GinzBurg, Le Sabbat des sorcières, Paris, 1992, p. 43-69.

17. S. SIMONSOHN, op. cit., vol. 7, p. 451-453. L. BARDINET, « La condition civile des Juifs du Comtat Venaissin pendant le séjour des Papes à Avignon », Revue historique 12, 1880, p. 1-47, et ID., «Les Juifs du Comtat Venaissin au Moyen Âge. Leur rôle économique et intellectuel ", Revue historique 14, 1880, p. 1-60, sp. p. 1-3. M. DE MAULDE, Les Juifs dans les États français du Saint Siège au Moyen Âge, Paris, 1886. A. MossÉ, Histoire des Juifs d'Avignon et du Comtat Venaissin, Marseille, 1976.

18. C. GinZBURG, op. cit., p. 78. 
ment vers le sabbat, en premier lieu la peur de nouvelles sectes et de la prolifération des pratiques de sorcellerie et de magie, ainsi que la mention des invocateurs de démons.

La bulle a certainement été rédigée sur la base des informations transmises par Ponce Feugeyron à Alexandre V ( $\ll \mathrm{j}$ 'ai entendu récemment que sur ces territoires, etc. »). N'est-ce pas sa propre phobie de nouvelles sectes qui transparaît dans la bulle, peur engendrée par les rapprochements bien réels entre les différentes communautés religieuses, dans le contexte très délicat et tendu du schisme? La bulle amplifierait ainsi des angoisses latentes.

Jamais un inquisiteur n'avait reçu un mandat aussi large, tant pour le nombre de tâches à effectuer que pour l'aire d'action définie dans la bulle d'Alexandre V ${ }^{19}$. Celle-là correspond à l'inquisition de Provence, placée entre les mains des franciscains depuis le XIVe siècle, et qui s'étend de la Méditerranée à la Savoie; elle est largement agrandie pour Ponce Feugeyron, puisque les diocèses de Lyon et de Belley y sont ajoutés. Le diocèse d'Aoste, appartenant à la Tarentaise, fait partie de ce ressort depuis son incorporation par Grégoire XI en 1375. La formule abrégée d' «inquisiteur d'Avignon » s'est naturellement imposée, puisque la résidence de l'inquisiteur est la ville des papes. Pour les juifs du diocèse d'Avignon et du Comtat Venaissin, placés sous autorité pontificale, la bulle implique un bouleversement dans les juridictions devant lesquels ils doivent être cités : ils passent de la juridiction du chambellan pontifical à Avignon ou de celle du recteur pontifical et de la cour temporelle s'ils résident dans le Comtat, à celle de l'inquisition. Par la suite, en 1418, Martin V rétablira la compétence d'un représentant du vicaire pontifical, précisément en raison des abus de l'inquisiteur ${ }^{20}$.

Pour un champ d'action aussi large, il faut des moyens considérables : quelques jours après la proclamation de la bulle, le 28 septembre, Alexandre V autorise Pierre de Thury, évêque de Maillezais, cardinal prêtre de Sainte-Suzanne et légat pontifical, à mettre à disposition de Ponce Feugeyron 300 florins par année pour couvrir ses dépenses et celles de son personnel. La somme est à payer par les juifs d'Avignon et du Comtat Venaissin. De plus, les ordinaires locaux doivent entretenir Ponce et ses hommes lors de leurs visites, ou leur donner 10 florins par

19. Soit « les provinces d'Arles. Aix et Embrun et tous les lieux situés à l'intérieur de ces dites provinces, ainsi que les diocèses de Lyon, Vienne, Belley, Grenoble, Maurienne, Die, Valence, Vivarais, Tarentaise et Aoste, et le Dauphiné, les comtats de Provence, Forcalquier et Venaissin, ainsi que la principauté d'Orange et le diocèse d'Avignon ».

20. J. M. VIDAL, op. cit., p. II-VII. S. SIMONSOHN, op. cit., vol. 7, p. 451-453. 
an ${ }^{21}$. Il y a là des moyens considérables pour mener de vastes campagnes, ouvrir des procédures, instituer et entretenir un personnel; cela implique une structure, basée à Avignon, et une organisation. De telles dépenses devaient être justifiées par un plan d'action. La nomination de Ponce Feugeyron à la charge d'inquisiteur n'est en aucun cas honorifique, comme c'est le cas dans d'autres régions au XVe siècle - notamment dans le royaume de France. Malheureusement, on sait peu de choses de son activité dans les premières années. On dispose tout de même d'informations significatives.

Tout d'abord, son mandat s'étend sur près de trente ans, puisque Martin V en 1418 et Eugène IV en 1435 confirmeront la bulle d'Alexandre $\mathrm{V}$ dans les mêmes termes, mais en supprimant la partie contre les schismatiques, qui a perdu de son actualité ${ }^{22}$. Nul besoin de ratifier un mandat qui reste lettre morte : on peut donc penser que Ponce Feugeyron s'est montré actif durant toutes ces années, comme en témoignent les informations recueillies ci-dessous.

En 1414, Jean XXIII ratifie la sentence d'acquittement prononcée par Ponce Feugeyron en faveur de Dominique Bens, laïc du diocèse de Grenoble, poursuivi à la requête de Jean Dupont et d'Antoine de Regiac, promoteurs de l'évêque de Grenoble et de l'inquisition ${ }^{23}$. Comme la personne incriminée réside dans les vallées vaudoises, il s'agit vraisemblablement d'un cas d'hérésie. En 1418, lorsque Martin V confirme la bulle d'Alexandre, le rayon d'action de l'inquisiteur s'étend au diocèse de Genève. Martin V tente de grignoter le ressort inquisitorial attribué aux dominicains ${ }^{24}$. Mais les dominicains ne se laissent pas faire et protestent contre cette extension du champ inquisitorial, qui est révoquée un an plus tard par Martin $\mathrm{V}^{25}$.

21. S. Simonsohn, op. cit., vol. 2, p. 660-661, doc. 584; ibid., vol. 7, p. 362-364.

22. S. SimONSOHN, op. cit., vol. 2, p. 667-669, doc. 590 (3 février 1418) et p. 824 827, doc. 705 (24 février 1435).

23. J. M. VIDAL, op. cit., p. 497-498.

24. Genève fait partie les diocèses dépendants de la province dominicaine de France, mais n'appartenant pas au royaume de France; il fait partie du lot qui est assigné aux dominicains par Nicolas IV en 1290. Voir J. M. VIDAL, op. cit., p. IV-V et B. ANDENMATTEN et K. UTZ TREMP, loc. cit., p. 71-73.

25. J. MARX, op. cit., p. 52. E. RiPOLL, Bullarium ordinis Fratrum Predicatorum, Rome, 1729-1740, t. 2, p. 566 sq. C. EUBEL, op. cit., p. 501, note au doc. 1371. Cette révocation sera suivie d'effet: en 1430 , les dominicains genevois s'en prennent à Baptiste de Mantoue, un prédicateur hétérodoxe, et sollicitent l'aide de l'inquisiteur Ulric de Torrenté pour mener l'enquête. Voir B. ANDENMATTEN et K. UTZ TREMP, loc. cit., p. 77-78 et L. BiNZ, «Les prédications hérétiques de Baptiste de Mantoue à Genève en 1430 », dans Pour une histoire qualitative. Études offertes à Sven Stelling. Michaud, Genève, 1975, p. 15-34. 


\section{Ponce Feugeyron et les juifs}

Contre les juifs, l'activité déployée par Ponce Feugeyron est manifeste; elle le conduit même à des excès que Martin V cherche à limiter en 1418 et 1421 : le pape le contraint à agir avec un représentant du vicaire pontifical, dans le respect du droit canon et non selon la procédure inquisitoire - l'accusé doit notamment pouvoir connaître le nom des témoins. Cela fait suite à des plaintes des juifs d'Avignon, qui auraient été à plusieurs reprises maltraités et molestés du fait des pratiques de l'inquisition. En effet, en 1419, la communauté d'Avignon, réunissant le conseil et le syndic de la ville, demande au pape d'accorder aux juifs de la cité d'Avignon la présence d'un représentant du vicaire pontifical à chaque fois que l'inquisiteur procède contre les juifs, pour les causes qui relèvent de l'inquisition. Une telle mesure n'aurait pas été nécessaire s'il n'y avait eu des abus et des plaintes, qui amènent la communauté chrétienne d'Avignon à marquer sa sollicitude et son soutien face à la communauté juive en adressant au souverain pontife une pétition décrivant à quel point les juifs d'Avignon ont été maltraités par Ponce Feugeyron. Rappelant le mandat d'Alexandre V de 1409, Martin V ordonne que François de Conzié, vicaire papal en Avignon et archevêque de Narbonne, attribue un représentant aux côtés de Ponce Feugeyron et de ses successeurs; Pierre Cottin, doyen de Saint-Pierre d'Avignon et chapelain, est désigné pour cette tâche. Ainsi, il apparaît nécessaire de contrôler l'inquisition dans son action contre les juifs en la faisant fonctionner avec un représentant pontifical. Malgré tout, cela ne suffit pas : en 1421, les juifs d'Avignon se plaignent auprès de Martin V de ce que le vicaire pontifical Pierre Cottin coopère avec l'inquisiteur contre eux; ils réclament aussi que les noms des témoins et des accusés leur soient communiqués. Martin $\mathrm{V}$ renouvelle alors les privilèges accordés par les prédécesseurs, notamment celui de Boniface VIII concernant le statut d'impotentes des juifs et celui de Grégoire XI, qui place les juifs, dans toutes les causes ne relevant pas de l'inquisition, sous la juridiction du viguier et des juges de la cour temporelle. Il enjoint Feugeyron et Cottin d'agir selon le droit canonique, et non selon le droit inquisitorial en rendant publics les noms des accusés et des témoins, et les menace d'excommunication en cas de désobéissance. Pour quelle raison l'inquisiteur a-t-il agi contre les juifs? On l'ignore pour l'heure, à part une brève allusion à l'usure dans la lettre de Martin V en 142126.

26. S. SimONSOHN, op. cit., vol. 2, p. 674-677, doc. 594, p. $700-709$, doc. 610-612 et ibid., vol. 7, p. 362-363. 
L'action de Ponce Feugeyron contre les communautés juives dépasse Avignon : en 1426, il mène dans les États de Savoie une importante campagne contre les livres juifs, en particulier contre le Talmud. Là, le but est clair : il s'agit de faire disparaître de la littérature hébraïque les passages jugés hérétiques ou erronés, en les biffant ou en les corrigeant, sur la base des conclusions que les juifs devaient abjurer et désavouer publiquement. À cette condition, les juifs peuvent utiliser leurs livres en toute tranquillité. Il leur en coûte toutefois 300 florins : on voit de quelle manière Ponce Feugeyron garantit sa provision annuelle! Le dossier, inédit mais brièvement résumé par Isidore Loeb qui consacrait son article à une enquête similaire conduite à Trévoux de $1429^{27}$, contient les articles abjurés par les juifs de Savoie, dont on peut penser qu'ils sont, en partie du moins, de la main de Ponce Feugeyron. L'affaire s'inscrit dans une tradition de condamnation de la littérature juive - pensons par exemple aux attaques de Pierre Le Vénérable (1092/1094-1156), dans son Adversus Iudaeos, contre le Talmud et la littérature rabbinique, et surtout à la condamnation et au brûlement du Talmud à Paris dans les années $1240^{28}$. Ici toutefois, les livres ne sont pas brûlés, mais soumis à la censure idéologique du dogme chrétien : on impose la suppression ou la correction des passages incriminés. Pour mener à bien sa tâche, Ponce Feugeyron n'agit pas seul : il collabore avec Guy Flamochet ${ }^{29}$, figure de proue, avec Jean Nider, du mouvement de réforme des Prêcheurs, et prieur du nouveau couvent dominicain de Chambéry - un couvent établi à proximité du quartier juif de la ville, ce qui laisse augurer de la qualité des relations de voisinage... Par ailleurs, cette affaire

27. I. LOEB, "Un épisode de l'histoire des Juifs de Savoie", Revue des études juives, tome $\mathrm{X}, \mathrm{n}^{\circ} 19-20$, janvier-juin 1885 , p. 32-59, sp. p. 50-54. Voir aussi Th. BARDELLE, Juden in einem Transit- und Brückenland. Studien zur Geschichte der Juden in Savoyen-Piemont bis zum Ende der Herrschaft Amadeus VIII, Hannover, 1998, p. 284-294.

28. D. IognA-PRAT, Ordonner et exclure. Cluny et la société chrétienne face à l'hérésie, au judaïsme et à l'islam. 1000-1150, Paris, 1998, p. 263-323; Y. FRIEDMAN, « Anti-Talmudic Invective from Peter the Venerable to Nicholas Donin (1144-1244)», dans Le Brûlement du Talmud à Paris : 1242-I244, G. DAHAN éd., Paris, 1999, p. 171 189; A. BoureaU, L'Événement sans fin. Récit et christianisme au Moyen Âge, Paris, 1993, p. 231-251.

29. Avant de se déterminer sur cette affaire, Amédée VIII délibère avec Ponce Feugeyron et cum prefato inquisitore prioreque predicatorum Chamberiaci, soit Guy Flamochet. À défaut des originaux turinois, j'ai consulté une copie conservée à la BnF, ms. lat. 12722, $f^{\circ} 105 r^{\circ}-108 r^{\circ}$, sp. $f^{\circ} 106 a$. En 1430, Guy Flamochet collaborera au grand procès intenté contre les vaudois de Fribourg, aux côtés d'Ulric de Torrenté; voir K. UTZ TREMP éd., Quellen zur Geschichte der Waldenser von Freiburg im Uechtland (1399-1439), Hannover, 2000 (MGH, Quellen zur Geschichte des Mittelalters, 18.Bd), p. 111. 
n'aurait pu être menée sans le soutien et la volonté politique du duc de Savoie Amédée VIII, qui voit sans doute à travers elle la possibilité de marquer son autorité et sa nouvelle dignité : le procès se tient peu de temps après l'élévation du comté de Savoie en duché (1416), dans une période où l'on assiste à la montée en puissance du duc. À l'instar de Thomas Bardelle, relevons le pragmatisme d'Amédée VIII qui condamne les juifs à une simple mais forte amende : un moyen de soulager les finances ducales tout en faisant la démonstration de son contrôle sur les communautés juives ${ }^{30}$.

\section{De Constance à Bâle}

En dehors de son activité contre les juifs et les hérétiques, Ponce Feugeyron est un personnage qui bénéfice d'un crédit important: il représente l'ordre des franciscains durant le concile de Constance (14141418); Martin V, lorsqu'il confirme la bulle d'Alexandre V à Constance en 1418 souligne «le zèle, la science, la sincérité et la maturité » de l'inquisiteur ${ }^{31}$. Rappelons que Constance est le concile de la fin du schisme et de la condamnation de Jean Hus. C'est d'ailleurs sur le thème de la restauration et de la réforme de l'Église et du clergé, ainsi que de l'importance du concile dans la fin du schisme, que Ponce Feugeyron prononce un sermon à Constance en $1417^{32}$.

On retrouve ensuite notre franciscain au concile de Bâle (14311449) : en 1433, il y est procureur du provincial des Mineurs pour la province de Provence et appartient également à une délégation de l'université d'Avignon. Il y prononce des sermons, malheureusement perdus ${ }^{33}$. Le 14 mars 1433, lors d'une session qui décide de l'admission des Hussites au concile, il se trouve à Bâle exactement en même temps que Georges de Saluces, nommé depuis peu évêque d'Aoste, avant d'être transféré à la tête du diocèse de Lausanne grâce aux bons soins du duc de Savoie Amédée VIII, dont il fut l'un des proches ${ }^{34}$. Or, c'est à Georges de Saluces que l'on doit précisément l'initiative des différentes chasses aux sorcières dans ces deux diocèses des États de

30. Th. BARDELLE, op. cit., p. 286-287, 294.

31. C. Eubel, op. cit., p. 501, n 1371, et S. SimONSOHN, op. cit., vol. 2, p. 667669 , doc. 590.

32. Acta Concilii Constanciensis, II : Konzilstagbücher, Sermoner, Reform- und Verfassungsakten, H. Fink éd., Münster, 1923, p. 513-516.

33. Concilium basiliense, Studien und Quellen zur Geschichte des Concils von Basel, J. HALleR éd., Bd. II, Basel, 1897, p. 513 (1 ${ }^{\text {cr }}$ novembre 1433).

34. Voir Concilium basiliense, op. cit., p. 369-370. La Visite des églises du diocèse de Lausanne en 1453, A. WILDERMANN éd. en collaboration avec V. PASCHE, Lausanne, 1993, vol. 1, p. 25-37. 
Savoie : ainsi, dans les statuts synodaux que l'évêque promulgue à Aoste en 1434, une année après son élection, il enjoint aux curés que soient dénoncés à la messe les hérétiques, les sorciers et les devins ${ }^{35}$. Des contacts entre les deux hommes à Bâle sont fort probables ${ }^{36}$; et l'on peut imaginer que l'inquisiteur, qui a poursuivi des juifs et qui vit dans l'angoisse de nouvelles sectes d'hérétiques juifs et chrétiens, de sorciers et d'invocateurs de démons, a pu trouver une oreille attentive auprès du réformateur Georges de Saluces, puisque celui-ci s'est montré dans sa carrière particulièrement sévère à l'égard des juifs et de tout ce qu'il considère comme hétérodoxe, hérétique et contre les bonnes mœurs.

Peut-être est-ce même pour cette raison qu'un an plus tard, Georges de Saluces fait alors appel à magister Poncius Frugeronis, soit Ponce Feugeyron, inquisiteur dans la cité et le diocèse d'Aoste, pour mener un procès de sorcellerie contre Mariette Caller, une femme de Valgrisenche ${ }^{37}$. L'inquisiteur, on s'en souvient, est autorisé à agir dans le diocèse d'Aoste par la bulle d'Alexandre $V$, qui sera renouvelée par Eugène IV l'année suivante, en février $1435^{38}$. Toutefois, Ponce Feugeyron n'agit pas lui-même au cours de cette affaire, mais délègue un chanoine d'Aoste, Pierre Magnin pour être juge et commissaire, et désigne comme son représentant ou vicaire le franciscain Martin de Cluse : l'inquisition est bien entre les mains des mineurs, avec le concours d'un ordinaire, en l'occurrence le chanoine d'Aoste. Une vaste enquête est menée dans les paroisses d'Avise, d'Arvier et de Valgrisenche. Ponce Feugeyron, à la tête d'une nouvelle structure, délègue ses compétences au gré des circonstances. De fait en 1439, Georges de Saluces dépose une requête au concile de Bâle pour pouvoir déférer à une ou plusieurs personnes le droit d'enquêter sur les affaires de foi, puisque aucun inquisiteur ne réside de manière permanente dans le diocèse d'Aoste ${ }^{39}$.

35. M. OSTORERO, "Folâtrer avec les démons". Sabbat et chasse aux sorciers à Vevey (1448), Lausanne, 1995 (CLHM, 15), p. 58-64. J. A. DuC, Histoire de l'Église d'Aoste, t. IV, Aoste, 1908, p. 378-379.

36. On peut même imaginer que les deux hommes se sont rencontrés une première fois à Avignon vers 1424-1426, car Georges de Saluces y étudie le droit canon ces années-là. Voir La Visite des églises du diocèse de Lausanne en 1453, op. cit., p. 26.

37. S. Bertolin, op. cit., p. 87 et 227 et F. GAMBA, « La sorcière de Saint-Vincent. Un procès d'hérésie et de sorcellerie au Xve siècle ", Bulletin de la Société académique, religieuse et scientifique du duché d'Aoste, 41, 1964, p. 283-311.

38. S. SiMONSOHN, op. cit., vol. 7, p. 824-826, doc. 705.

39. Concilium Basiliense, Studien und Quellen zur Geschichte des Concils von Basel, G. BECKMANN éd., t. VI, Basel, 1926, p. 655. La même année, Jean Andrée (futur officiel de Lausanne) est délégué par Ponce pour instruire le procès de Barthélemy Bertaca. 
Enfin c'est en liaison avec le concile de Bâle que l'on retrouve des manuscrits des Errores gazariorum : le texte contenu dans le codex de la Bibliothèque Vaticane (Vat. Lat. 456) est inséré, dans un résumé chronologique des sessions du concile de Bâle, entre la $24^{\mathrm{e}}$ session de mai 1436, elle-même suivie d'une copie d'une lettre datée de mars 1437, et la $25^{\mathrm{e}}$ session de mai 1437 ; le manuscrit de la Bibliothèque universitaire de Bâle (A II 34) contient quant à lui différents actes liés au concile, et en particulier les négociations avec les Hussites ${ }^{40}$.

\section{Ponce Feugeyron, auteur des Errores gazariorum?}

Vu sous cet angle, n'apparaîtrait-il pas possible que Ponce Feugeyron soit l'auteur des Errores? Dans ce cas, le texte serait le fruit d'un travail de maturité et constituerait le bilan de ses actions : quand il le rédige, Ponce Feugeyron est inquisiteur depuis près de 30 ans, il doit avoir une soixantaine d'années, mais il est encore actif, puisqu'Eugène IV confirme la bulle d'Alexandre V en 1435. Riche de trente ans d'expérience, il rédigerait son texte probablement au moment où est mis en place un tribunal d'inquisition à Aoste, avec sa collaboration, et nécessairement après 1428, année de l'exécution de Jeannette Cauda, mentionnée dans les Errores gazariorum. Ponce aurait ensuite trouvé dans le concile de Bâle, où il se rend dans les années 1433-1437, le milieu de diffusion idéal. Rappelons que plusieurs personnages dont les noms sont liés aux premières chasses aux sorciers sont présents à Bâle dans ces mêmes années : l'évêque Georges de Saluces, le dominicain Jean Nider, qui raconte dans son Formicarius les procès du Simmental et ceux du diocèse de Lausanne, l'inquisiteur et auteur du Flagellum hereticorum fascinariorum Nicolas Jacquier, ainsi que le doyen du chapitre de Cambrai Gilles Carlier, consulté lors de la Vauderie d'Arras en 1460.

Après les sectes de juifs et de chrétiens comploteurs et sorciers de 1409, Ponce Feugeyron a trouvé un nouveau danger à stigmatiser, plus invisible, et par conséquent plus menaçant. Son texte se ferait l'écho de ses propres angoisses : les sectes, les alliances entre différentes communautés sous l'égide du diable, et bien sûr la sorcellerie. Personnage de son temps, qui vit dans le fantasme des sectes occultes - pensons à Laurent Pignon et à sa secte de devineurs ${ }^{41}$ - , il a partiellement contri-

40. M. OSTORERO et alii, op. cit., p. 269-275.

41. J. VÉRONĖSE, « Jean sans Peur et la "fole secte" des devins : enjeux et circonstances de la rédaction du traité Contre les devineurs (1411) de Laurent Pignon », Médiévales, 40, printemps 2001, p. 113-132. J. R. VEENSTRA, Magic and Divination at the Courts of Burgundy and France. Text and Context of Laurens Pignon's Contre les devineurs (/4II), Leyde-New York-Cologne, 1998. 
bué à donner forme et à amplifier un nouveau danger. Feugeyron n'est certainement pas un cas exceptionnel : dans le contexte du schisme et de la crise de l'Église, il partage avec d'autres, en particulier parmi l'élite, la peur des sectes et des déviances hérétiques ou hétérodoxes, et surtout la peur des « mélanges » - réels ou imaginaires : entre juifs et sorciers, entre juifs et hérétiques, entre hérétiques et sorciers. C'est bien dans ce contexte que va émerger le fantasme du sabbat.

Enfin, n'oublions pas que le mandat d'inquisiteur de Ponce Feugeyron couvre également la région du Dauphiné. Si l'on accepte qu'il ait rédigé les Errores gazariorum, on pourrait ainsi comprendre pourquoi la vision du sabbat développée par Claude Tholosan, juge-mage laïc du Dauphiné, est si proche de celle des Errores gazariorum ${ }^{42}$. Les deux hommes ont peut-être été amenés à collaborer lors des procès intentés contre les vaudois ou les sorciers. Pour l'heure, nous n'avons pas retrouvé de mention de Ponce Feugeyron en Dauphiné; d'autres inquisiteurs ou vice-inquisiteurs franciscains (Pierre Fabri, Antoine André, Guillaume de Malavieille, etc.) y sont attestés. Toutefois, en vertu de l'étendue du mandat de Feugeyron et de son importante provision annuelle de 300 florins, on peut se demander s'il n'a pas contribué à installer des inquisiteurs agissant localement sur un territoire; il serait alors le grand orchestrateur de l'inquisition, dans un territoire qui va de la Méditerranée au val d'Aoste en passant par les Alpes occidentales, dans les années clés de la lutte contre les hérésies et le début de la formation de l'imaginaire du sabbat.

En retraçant l'itinéraire de Ponce Feugeyron, de la bulle de 1409 jusqu'au concile de Bâle, on saisit toute l'importance des juifs dans la création de l'imaginaire du sabbat des sorcières. Sur ce plan, c'est l'hypothèse de Carlo Ginzburg relative au fantasme du complot qui se trouve renforcée, mais d'une manière quelque peu différente. Si l'on admet que Ponce pourrait être l'auteur des Errores gazariorum, les traits d'anti-judaïsme que j'avais cru y percevoir s'expliquent d'autant mieux ${ }^{43}$. D'ailleurs, le texte des Errores ne se rapproche-t-il pas, sous certains aspects formels et thématiques, des pamphlets anti-juifs ? Quoi qu'il en soit, que l'on fasse ou non de Feugeyron l'auteur des Errores gazariorum, on ne peut désormais nier qu'il est en tout cas très proche des premières élaborations du sabbat. A ce titre, son itinéraire et sa

42. Voir P. PARAVY, «À propos de la genèse médiévale des chasses aux sorcières : le traité de Claude Tholosan, juge dauphinois (vers 1436) », Mélanges de l'École française de Rome. Moyen Âge/Temps modernes, 91, 1979, p. 333-379, repris dans M. OSTORERO et alii, op. cit., p. 357-438.

43. M. OSTORERO et alii, op. cit., p. 323-327. 
carrière méritent une grande attention, cela d'autant plus que notre personnage évolue dans la région clé de l'arc alpin occidental et du duché de Savoie, lieux d'émergence des premières chasses aux sorciers.

Martine Ostorero, Université de Lausanne (FNRS), Section d'histoire médiévale BFS H2, CH-1015 Lausanne

Itinéraire d'un inquisiteur gâté : Ponce Feugeyron, les juifs et le sabbat des sorciers

Dans la première moitié $d u X v^{e}$ siècle, l'inquisiteur franciscain Ponce Feugeyron est mandaté par le pape Alexandre $\mathrm{V}$ pour agir tant contre les juifs et les judaïsants que contre les sectes d'hérétiques et de sorciers. Son rayon d'action couvre tout le Sud-Est de la France, d'Avignon au duché de Savoie en passant par le Dauphiné. On examine ici l'hypothèse qu'il pourrait être l'auteur des Errores gazariorum, l'un des plus anciens textes présentant l'imaginaire du sabbat, écrit dans le val d'Aoste vers 1436.

Inquisition - inquisiteur - sorcellerie - hérésie - juifs - Ponce Feugeyron

Itinerary of a spoiled inquisitor : Ponce Feugeyron, the Jews and the witches' sabbath

A Franciscan living in the first half of the fifteenth century, Ponce Feugeyron was an inquisitor whose activities spread over Jews, heretics and witches. He covered an area wich included nearly all of Southeastern France, from Avigon throught the Dauphiné to the duchy of Savoy. He might also be the author of one of the very first texts mentioning the witches' sabbath, namely Errores gazariorum (errors of the « Gazari »), an anonymous treatise written in the Aosta Valley around 1436.

Inquisition - inquisitor - witchcraft - heresy - Jews - Ponce Feugeyron 